\section{SPIRO - the automated Petri plate imaging platform designed by biologists, for biologists}

Jonas A Ohlsson ${ }^{1 *}$, Jia Xuan Leong ${ }^{2,3 *}$, Pernilla H Elander ${ }^{1}$, Adrian N Dauphinee ${ }^{1}$, Florentine Ballhaus ${ }^{1}$, Johan Johansson ${ }^{4}$, Mark Lommel ${ }^{2}$, Gero Hofmann ${ }^{2}$, Staffan Betnér ${ }^{5,6,7}$, Mats Sandgren ${ }^{1}$, Karin Schumacher ${ }^{2}$, Peter V Bozhkov $^{1}$ and Elena A Minina ${ }^{1,2^{*}}$

${ }^{1}$ Department of Molecular Sciences, Uppsala BioCenter, Swedish University of Agricultural Sciences and Linnean Center for Plant Biology, P.O. Box 7015, Uppsala, SE-750 07, Sweden

2 Centre for Organismal Studies (COS), Heidelberg University. Im Neuenheimer Feld 230. 69120 Heidelberg, Germany

${ }^{3}$ Center for Plant Molecular Biology (ZMBP), University of Tübingen, Auf der Morgenstelle 32. D-72076 Tübingen, Germany

${ }^{4}$ Johan's 3D printing service, Uppsala, Sweden

${ }^{5}$ Centre for Occupational and Environmental Medicine, Stockholm County Council, Stockholm, Sweden.

${ }^{6}$ Department of Energy and Technology, Division of Applied Statistics and Mathematics, PO Box 7032, Swedish University of Agricultural Sciences, SE75007 Uppsala, Sweden.

${ }^{7}$ Institute of Environmental Medicine, Karolinska Institutet, SE-171 77, Stockholm, Sweden.

${ }^{*}$ For correspondence:

EAM: alena.minina@slu.se

JAO: jonas.ohlsson@slu.se

JXL: jia-Xuan.leong@zmbp.uni-tuebingen.de 


\section{Abstract}

25 The imaging of plant seedlings, fungal mycelia and bacterial colonies grown

26 on Petri plates is common in phenotyping assays, and is typically done

27 manually despite the procedures being time-consuming and laborious. The

28 main reason for this is the still limited availability of existing automated

29 phenotyping tools and facilities. Additionally, constructing a custom-made

30 automated solution is a daunting task for most research groups specializing in

31 biology.

32 Here, we describe SPIRO, the Smart Plate Imaging Robot, an automated

33 platform that acquires time-lapse photos of up to four vertically oriented Petri

34 plates in a single experiment. SPIRO was designed for biologists by biologists;

35 thus, its assembly does not require experience in engineering or programming

36 and its operation is sufficiently intuitive to be carried out without training.

37 SPIRO has a small footprint optimal for fitting into standard incubators for

38 plants and microbes, and is equipped with an LED light source for imaging in

39 the dark, thus allowing acquisition of photos under optimal growth conditions.

40 SPIRO's web-based user interface allows setting up experiments and

41 downloading data remotely, without interfering with samples growth.

42 SPIRO's excellent image quality is optimal for automated image processing,

43 which we demonstrate with two semi-automated assays for analysis of

44 commonly used phenotypic traits: seed germination and root growth.

45 Moreover, the robot can be easily customized for a specific use, as all 46 information about SPIRO, including the models for 3D-printed structural

47 components, control software, and scripts for image analysis, are released

48 under permissive open-source licenses.

49 Keywords: Time-lapse imaging, Phenotyping, Arabidopsis, Roots, Root 50 growth, Seeds, Germination, Automated imaging, Automated image analysis, 51 ImageJ, ImageJ macro, R, Raspberry Pi, 3D printing, Open science hardware,

52 Lab automation 


\section{Introduction}

54 Manual imaging of Petri plates using cameras or scanners is a common

55 practice in biology experiments that require phenotyping of plant seedlings or

56 microbial colonies. However, manual imaging necessitates removing the

57 plates from the growth conditions and increases the risk of introducing

58 unwanted variables into the experiment, e.g., changes in temperature,

59 humidity, illumination, vector of gravity, and mechanical stress. Such

60 fluctuations, especially if triggered repeatedly during time-lapse experiments,

61 might significantly impact the phenotypes of interest, including plant seed

62 germination, root growth, or microbial colony growth ${ }^{1-3}$. Furthermore, manual

63 imaging has limited time resolution, causes inconsistencies in the time of

64 imaging and impedes data acquisition during nights.

65 Automating labor- and time-intensive procedures is crucial to improving

66 research quality and throughput, and open science hardware and software

67 (tools which are freely available and modifiable) can help further this goal ${ }^{4-6}$.

68 Moreover, open source hardware improves the transparency and

69 reproducibility of science while delivering radical cost savings ${ }^{6}$, enabling less

70 well-funded labs (including those in low-income countries) to afford high-

71 quality equipment ${ }^{5}$.

72 A plethora of commercial and custom-made automated systems for imaging

73 biological samples on Petri plates are already available ${ }^{7-13}$. However, we

74 struggled to find an affordable platform that would be suitable for imaging

75 Petri plates in standard plant growth incubators. Performing automated

76 imaging under the growth conditions used for other experiments is crucial for

77 direct comparison of the results, therefore we endeavored into developing a

78 custom-made small footprint solution.

79 The result of our efforts is SPIRO, the compact Smart Plate Imaging Robot

80 for time-lapse imaging of vertical Petri plates, which fits into standard

81 plant/microbe incubators. SPIRO was designed for biologists by biologists,

82 introducing end-user insight into its development. We ensured that no prior

83 knowledge of mechanical engineering, electronics, or computer science is

84 necessary for its assembly and operation. SPIRO comprises the absolute 
85 minimum of components that warrants robust and reliable high-throughput

86 time-lapse imaging and applicability for a broad range of experimental

87 layouts. Owing to its minimalistic design, building the robot costs less than

$88 € 200$ (as of 2019), and it is not only easy to assemble but also to maintain,

89 making it optimal for every-day use.

90 To further promote SPIRO's applicability, we have developed two designated

91 assays for high-throughput analysis of images produced by the robot: SPIRO

92 Seed Germination and SPIRO Root Growth Assays. The assays are designed

93 for analysis of phenotypic traits commonly used in plant biology: seed size,

94 germination time, primary root length and growth rate. SPIRO assays

95 encompass complete start-to-finish detailed procedures comprising the

96 preparation of Petri plates, automated imaging under user-defined conditions,

97 semi-automated image processing and statistical analysis of the quantitative

98 data.

99 SPIRO is powered by the open source computer platform Raspberry $\mathrm{Pi}^{14}$ and

100 comprises mostly 3D-printed hardware components, making it particularly

101 suitable for customization. For the benefit of the scientific community, we are

102 publishing SPIRO as an open source project with all information about its

103 structural design, electronics, software and designated assays available under

104 permissive licenses allowing unlimited use and modifications in the presence

105 of correct attribution. 
108 SPIRO takes 8 megapixel (MP) time-lapse images of up to four Petri plates

109 positioned on a rotating cube-shaped stage. It is equipped with green LEDs for

110 illuminating plates while imaging in the dark, and is controlled via a web-

111 based user interface (Fig. 1A-D, Movie S1). The latter feature enables setting

112 up imaging conditions remotely via an Ethernet cable, Wi-Fi, or a built-in Wi-

113 Fi hotspot, while the robot is inside a growth cabinet. (Fig. 1A) SPIRO's

114 dimensions are ca $50 \mathrm{~cm} \times 30 \mathrm{~cm} \times 30 \mathrm{~cm}$ (length $\times$ width $\times$ height), it weighs

115 less than $3 \mathrm{~kg}$, and can easily be moved between locations.

116 SPIRO performs imaging in cycles, where one cycle comprises: (i) finding the

117 "home" position, at which the first plate holder is facing the camera; (ii)

118 measuring the average light intensity to determine if the ambient light intensity

119 is sufficient for image acquisition without green LED illumination, or

120 otherwise switching the LEDs on; (iii) taking an image and saving the file; (iv)

121 rotating the stage by $90^{\circ}$ to place the next plate holder in front of the camera,

122 and repeating steps ii and iii until all plates are imaged. The duration of each

123 cycle is less than two minutes, enabling high temporal resolution time-lapse

124 imaging.

\section{$125 \quad$ Hardware design}

126 The three main goals of the SPIRO hardware design were that it should be

127 affordable, customizable, and that a person with no previous experience could

128 build it easily. For this reason, we opted to use 3D-printed parts and standard

129 aluminium profiles for the structural components (Figure 1, Tables S1 and

130 S2, Files S1 and S2, and the SPIRO Hardware Repository ${ }^{15}$ ), and relatively

131 cheap and readily available electronic components. 3D printing is inexpensive,

132 allows for reproducible fabrication, rapid prototyping and modification, and is

133 easily accessible. For example, printing can be done at publicly available

134 facilities such as Makerspaces ${ }^{16}$ or ordered from online services (3dhubs ${ }^{17}$ or

135 similar). Printed parts can be easily replicated if they get broken or customized 
136 for a specific application. We successfully printed and tested four SPIRO

137 prototypes in two independent laboratories using black matte PLA filament

138 (for detailed information about printing, see Table S2 and the SPIRO

139 Hardware Repository ${ }^{15}$ ). The hardware proved to be easy to reproduce, robust

140 and durable.

141 To facilitate use of SPIRO for a broad range of experiments we designed plate

142 holders compatible with the most commonly used plate formats: a $12 \mathrm{~cm}$

143 square (Greiner Bio-One International, Item: 688102) and a $9 \mathrm{~cm}$ round Petri

144 plate (Sarstedt, 82.1473.001), and enabled adjusting distance between the

145 camera and the plate holders by moving the camera along the vertical and

146 horizontal aluminum profiles (Fig. 1A).

147 Camera

148 SPIRO is equipped with a single $8 \mathrm{MP}(3280 \times 2464$ pixels $)$ color camera, and 149 saves images as RGB PNG files. Image files are stored in a user-defined 150 experiment folder and are automatically sorted into four sub-folders 151 corresponding to each plate holder. Metadata useful for further analysis is 152 included into the file names, i.e., the names contain the plate holder number, 153 date and time of acquisition, and information about day or night mode (for 154 detailed information, please refer to File S4). SPIRO acquires excellent quality 155 images regardless of ambient illumination conditions, which is crucial for 156 downstream automated data analysis (Fig. 1E and F, Movie S2).

157 We provide assembly instructions for two possible configurations of SPIRO 158 (GitHub SPIRO Hardware ${ }^{15}$, File S2): the first is based on a Raspberry Pi v2 159 camera and requires manual adjustment of the focus before starting an 160 experiment; the second one implements an Arducam camera module with motorized focus, which enables remote focusing via the SPIRO software. Both cameras are very compact and allow imaging of complete Petri plates from a short distance without fisheye distortion effects, a feature crucial for quantitative comparison of seed and seedling measurements. In our experience, both configurations deliver the same image quality, and while the first configuration is somewhat cheaper, the second one is more convenient. 
167 Notably, the first configuration can be relatively easily upgraded into the

168 second. Furthermore, SPIRO is compatible with a range of MPI CSI (Mobile

169 Industry Processor Interface Camera Serial Interface) cameras, which

170 implementation might require minimal modifications of the camera house. As

171 new cameras are being continuously developed, we strongly recommend

172 checking the SPIRO Hardware repository for potential upgrades.

\section{Computer}

174 SPIRO's electronics layout (File S2) is optimized to enable all essential

175 features for robust high-throughput imaging while minimizing costs and

176 assembling complexity. SPIRO is powered by the cheap and readily available

177 Raspberry Pi 3B + single-board computer ${ }^{14}$ that controls the other four

178 components: a camera, stepper motor, positional sensor (mini microswitch)

179 and LED light source. SPIRO's software and acquired images are hosted on a

180 microSD card mounted on the Raspberry Pi computer and can be remotely

181 accessed via Ethernet or Wi-Fi connection. Notably, Raspberry Pi is an open

182 source computer platform designed for development of engineering solutions

183 and is supported by a vivid community. Raspberry Pi is compatible with a

184 multitude of electronics modules, sensors and components, often

185 supplemented with suitable software packages ${ }^{18}$. It is thus optimal for further

186 customizing the current SPIRO layout for specific uses.

187 Stepper motor and positional sensor

188 The cube-shaped stage of SPIRO is rotated by a stepper motor (i) during 189 imaging, to position each of the four plate holders in front of the camera and

190 (ii) between the imaging cycles, to ensure the plates are evenly exposed to the 191 ambient conditions and that there is no shading effect from SPIRO's light 192 screen (Fig. 1).

193 The $12 \mathrm{~V}$ unipolar stepper motor we recommend provides sufficient force to 194 reproducibly move the weight corresponding to the stage with 4 square Petri 195 plates containing medium and plants and a holding torque that stably holds the 196 stage position during imaging. Importantly, the motor movement is smooth 
197 and has no impact on Arabidopsis root growth under normal conditions

198 (Movie S2).

199 The current layout of SPIRO requires two power supply units: a 5 V supply

200 for the Raspberry Pi computer and a 12 V supply for the stepper motor and

201 LED illuminator (Fig. 1A, Table S1, File S2). We decided for such

202 configuration, as it drastically simplifies the assembly and maintenance of the

203 robot, in comparison to implementing a single power supply unit.

204 At the beginning of each imaging cycle, the motor rotates the stage until the

205 pin attached to the bottom of the stage presses the lever of a positional

206 microswitch (Fig. 1A, Movie S1). After the signal from the microswitch is

207 detected, the stepper motor makes a predefined number of steps, thus placing

208 the first plate holder in front of the camera. If needed, the number of steps can

209 be adjusted by the user in the "Calibrate motor" settings tab of the SPIRO

210 software. After the image of the first plate holder is taken, the motor rotates

211 the stage by $90^{\circ}$ three more times, pausing to acquire images of the other three

212 plate holders of SPIRO.

213 During prototyping, we considered implementation of either magnetic or 214 infrared (IR) switches. However, a mechanical sensor provides the most robust

215 system that is least susceptible to the presence of magnetic fields or stray light

216 in the environment, and thus is applicable to a broader range of growth

217 cabinets.

218 LEDs

219 SPIRO's built-in light source enables imaging of Petri plates in the dark, 220 providing another crucial benefit over manual imaging. The light source 221 comprises a green LED strip mounted on a 3D-printed square frame with a 222 diffuser (Figure. 1, Movie S1). The LED frame can slide along the horizontal 223 axis to fine-tune illumination of the Petri plates for individual conditions (Fig. 224 1A). 
225 SPIRO does not require any instructions from the user about the day/night

226 cycles in the growth cabinet. The intensity of ambient illumination is

227 automatically assessed by SPIRO's camera immediately prior to acquiring

228 each image. If sufficient illumination is detected, SPIRO takes an image in the

229 "day" mode (Fig. 1C, E), otherwise the robot turns on the LED light source

230 and acquires a "night" image (Fig. 1D, F). ISO and shutter speed for image

231 acquisition can be adjusted individually for day and night modes in the web-

232 based interface of the SPIRO software (Fig. 1B).

233 During prototyping, we tested night imaging using IR LEDs and the IR-

234 sensitive Raspberry Pi NoIR camera. However, this increased the cost of the

235 robot, while significantly complicating its electronics layout and focusing

236 procedure, and did not provide satisfactory quality of images suitable for

237 automated image analysis.

238 Typically, color camera detectors are most sensitive to green light, as they

239 contain double the number of green pixels compared to red or blue. Hence, we

240 speculated that using a green light source for illumination would be most

241 efficient while using the color Raspberry Pi camera for imaging in the dark.

242 Additionally, we took into consideration use of SPIRO for plant imaging.

243 Plants are known to be dependent on the light of blue and red wavelengths for

244 photosynthesis and regulation of the circadian cycle ${ }^{19,20}$. Although a number

245 of studies showed that green light wavelengths also have important regulatory

246 effect during plant growth and development, the reported effects were

247 observed after prolonged irradiation ${ }^{21}$. Thus, we speculated that illuminating

248 Petri plates with green LEDs only during imaging, should have the weakest

249 impact on the growth and circadian cycle of imaged seedlings. To verify

250 whether this was the case, we compared germination rates and root growth of

251 Arabidopsis thaliana seeds and seedlings, respectively, imaged using two

252 SPIRO systems, each with and without green LEDs. Germination was

253 assessed using an ANOVA test where germination rate at $t=50 \mathrm{~h}$ was set as

254 the dependent variable. Germination rate was compared at a single time point

$255(50 \mathrm{~h})$ due to the constraints imposed on germination detection by not having

256 night image data available. For comparing the effect of LED illumination on

257 root growth, plates with identical genotypes and media were imaged with and 
258 without LED illumination. Two models were fitted: the first was the standard

259 root growth model as described in the SPIRO Assay Manual (File S4), and the

260 second was the same model but with the fixed effect of LED and all of its

261 interaction effects added. Models were then compared using the anova

262 function in R. Our analysis confirmed that green light indeed had no effect on

263 germination and root growth (Tables 1 and 2).

264 Table 1. The results of an ANOVA test with one dependent variable 265 (Germinated before $t=50 \mathrm{~h}$ ), and three independent variables (genotype, 266 system, LED), using four genotypes and 1154 seeds, indicate that SPIRO 267 systems do not differ in performance and that LED illumination does not 268 influence germination rates. The experiment was performed using the 269 protocol provided in File $\mathbf{S 4}$.

\begin{tabular}{lllllll}
\hline & D.F. & Sum Sq & Mean Sq & F-value & p-value \\
\hline Genotype & 3 & 17.15 & 5.716 & 24.723 & $\begin{array}{l}1.67 \\
10^{-15}\end{array}$ & $\times$ \\
System & 1 & 0.09 & 0.094 & 0.407 & 0.524 \\
LED & 1 & 0.55 & 0.545 & 2.357 & 0.125 \\
Residuals & 1148 & 265.44 & 0.231 & & \\
\hline
\end{tabular}

Table 2. Results of the comparison of root growth models with and without 272 effect of LED, using 316 seedlings on two different SPIRO systems. The better 273 model is the one not including the effects of LED, indicating that LED 274 illumination has no effect on root growth

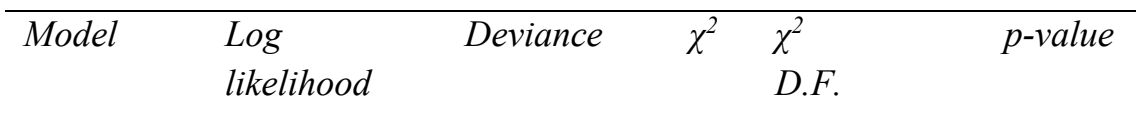

\begin{tabular}{llllll}
\hline $\begin{array}{l}\text { Without } \\
\text { LED }\end{array}$ & 65440 & -130880 & & & \\
With LED & 65281 & -130563 & 0 & 30 & 1.00 \\
\hline
\end{tabular}


A
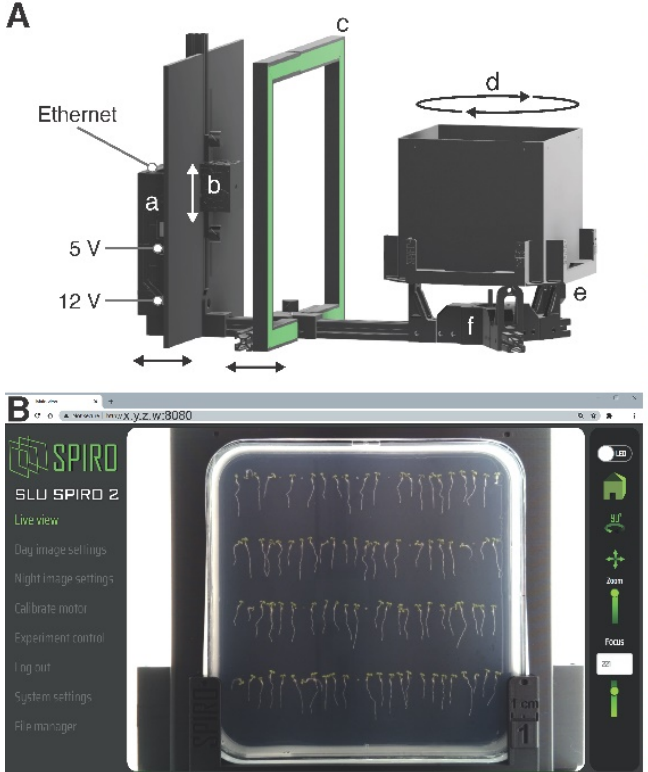
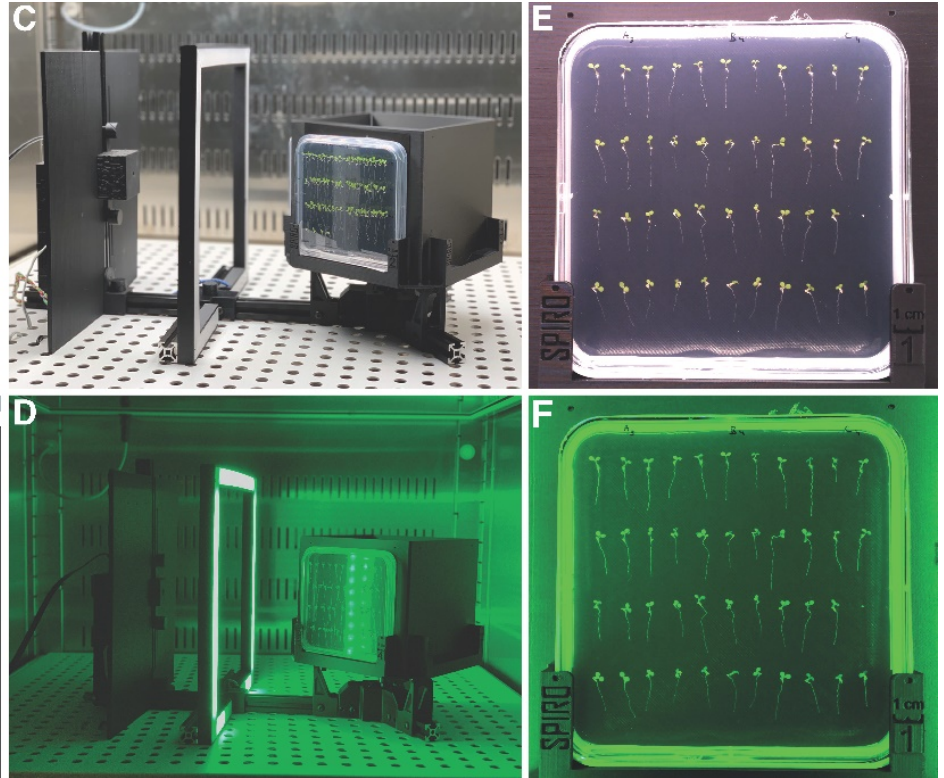

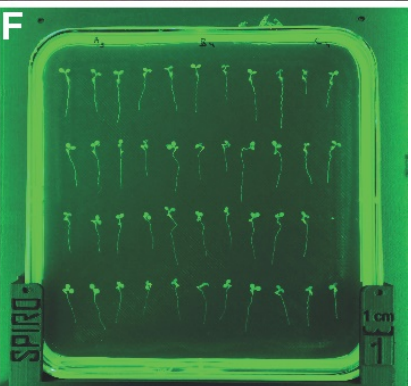

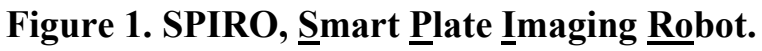

(A) 3D rendering image of SPIRO. The robot is controlled by a Raspberry Pi computer placed within the electronics housing (a). The camera house is mounted on a vertical axis with an anti-reflection screen (b). The white arrow indicates possibility of adjusting the camera position along the vertical axis, the black arrows indicate the possibility to tune the distance between the camera and the stage. A green LED frame (c) provides illumination during imaging under night conditions. The cube-shaped stage can accommodate up to four Petri plates (d). At the beginning of each imaging cycle, the home position of the stage (where the first plate holder is facing the camera) is verified with the help of the positional sensor (e). The stage is rotated by a stepper motor (f).

(B) SPIRO is controlled by the designated software via a web-based graphical user interface, which allows users to adjust the settings for an experiment and to access imaging data.

(C) SPIRO in a plant growth cabinet under day conditions

(D) SPIRO in a plant growth cabinet under night conditions. SPIRO automatically detects insufficient illumination and turns on the LED for a fraction of a second while the image is taken.

(E, F) Examples of images acquired by SPIRO under day and night conditions, respectively. 


\section{SPIRO Accessories}

278 To aid the use of SPIRO we designed a set of essential accessories (Table S2

279 and the SPIRO Hardware GitHub Repository ${ }^{15}$ ).

280 3D-printable seed plating guides help the user to place Arabidopsis seeds on 281 plates at regular distances from each other and from the edges of the plate. The 282 latter is important to avoid overlaying seed and seedling images with 283 reflections and shadows caused by the Petri plates' rims. The marked regular 284 distance positions for seeds were optimized for the SPIRO Germination and

285 Root Growth Assays described below.

286 The anti-reflection lids are designed to reduce reflections from seeds and 287 seedlings that are usually visible in the Petri plates' lids. Although such 288 reflections might not be an issue during imaging, their presence is detrimental 289 for automated image processing, as some of them are difficult to automatically 290 differentiate from actual biological samples (for more information see Fig. 2 291 in the File S4).

292 Assembling SPIRO

293 SPIRO was developed specifically to enable its assembly by a person with no 294 expertise or training in engineering, electronics and 3D printing. The complete 295 list of components, step by step instructions, and tutorial videos for assembly 296 are provided in Tables S1 and S2, and Files S1 and S2. However, we highly 297 recommend to check the SPIRO Hardware Repository ${ }^{15}$ for potential updates 298 before assembly. SPIRO hardware includes a set of standard parts, like aluminium profiles, screws and electronic components that need to be purchased. The complete list of these components and links with suggestions on where to purchase them is provided in Table S1 and the SPIRO Hardware Repository ${ }^{15}$. Our experience of ordering hardware to build SPIRO prototypes in Germany and Sweden reproducibly showed that the most challenging part to acquire are the correct screws. At the moment, we cannot provide a plausible explanation for 
306 this peculiar phenomenon and will try to upgrade the SPIRO specifications to

307 reduce the requirements for screws. Notably, approximately one quarter of

308 purchase costs were covering shipping expenses. Furthermore, some parts,

309 such as the LED strips, had to be ordered in excess. Thus, building several

310 SPIROs lowers the price per robot.

311 The STL, 3MF and F3D files for 3D-printable parts of SPIRO and the printing 312 settings recommendations are provided in Table S2, File S2 and the SPIRO

313 Hardware Repository ${ }^{15}$. SPIRO's hardware was tested and optimized to be

314 printed using PLA (polylactic acid) filament, which is the least expensive and

315 sufficiently robust type of printable plastic. Printing in PETG (Polyethylene

316 terephthalate glycol) and ABS (Acrylonitrile butadiene styrene) plastic is

317 technically possible, but would require adjustment in scaling and printing

318 settings, as the printed parts might shrink or warp significantly.

319 Printing all SPIRO parts using one 3D printer takes about seven days. The 320 prototyping was done using Prusa i3 MK2/S and MK3S printers. Nevertheless, 321 the pre-arranged components sets we provide (Table S2, SPIRO Hardware 322 Repository $^{15}$ ) can be printed on any type of $3 \mathrm{D}$ printer with the printing 323 volume of $25 \times 21 \times 20 \mathrm{~cm}$ or more. In our experience, the assembly procedure 324 can be completed by a determined untrained person in approximately two full325 time work days, while an experienced user can assemble SPIRO in about four 326 hours.

\section{Software and Installation}

328 Since SPIRO was designed to be used within plant growth cabinets, we 329 developed software that allows the remote control of SPIRO via the internet.

330 Besides convenience of use, remote control of the robot is essential to enable setting up imaging parameters under the conditions that will be used during the experiment. SPIRO's software is used for adjusting the camera focus, setting up ISO and shutter speeds for day and night imaging conditions, defining the name and the duration of an experiment and the frequency of imaging, starting and terminating experiments and downloading imaging data 
336 (for the detailed information please refer to File S4 and the SPIRO Software

337 Repository $\left.{ }^{22}\right)$.

338 SPIRO's software has an intuitive web-based graphical user interface that can

339 be easily accessed from any web browser (Fig. 1B). The layout of the software

340 was optimized with the help of several beta-testers to ensure that the interface

341 is sufficiently self-explanatory, does not require training prior to use and

342 contains the complete minimal number of essential features. The program and

343 detailed installation instructions are provided in File S2 and the SPIRO

344 Software Repository ${ }^{22}$. The installation procedure requires SPIRO to be

345 connected to the network, which can be done via the Ethernet port of the

346 Raspberry Pi computer (Fig. 1A) or by connecting to a Wi-Fi network. For

347 convenience, we recommend assigning SPIRO a static IP number, if this is

348 possible within the user's network. After installation is complete, it is possible

349 to activate a Wi-Fi hotspot from SPIRO's Raspberry Pi computer and use it

350 for future connections to the robot (for instructions see File S2 and the SPIRO

351 Software Repository ${ }^{22}$ ).

352 While setting up and starting a SPIRO experiment is done via an internet 353 connection or Wi-Fi hotspot, running the experiment does not require the robot

354 to be online. However, internet connectivity provides access to images during

355 the experiment run and to the data from the previously completed experiments.

356 The SPIRO software is written in Python 3 and released under an open source

357 2-clause Berkeley Software Distribution (BSD) license, allowing

358 redistribution and modification of the source code as long as the original

359 license and copyright notice are retained. SPIRO's simple and versatile

360 program for image acquisition includes features making automated imaging

361 possible under conditions that might vary between experiments and

362 laboratories:

- Imaging cycles are carried out at a user-defined frequency and duration. Before each imaging cycle, the stepper motor makes a predefined number of steps after the positional microswitch sensor was detected in order to place the stage in the "home" position. 
- Image acquisition is preceded by assessment of illumination intensity. If the average pixel intensity on a sampled image is less than 10 (out of a maximum of 255), the software triggers acquisition under userdefined "night" settings and the LEDs are switched on for the duration of acquisition (less than one second). If the average pixel intensity on the sample image is higher than 10, the image is acquired with userdefined "day" settings.

- Full resolution RGB photos of each of the four plate holders are saved as PNG files on the microSD card mounted on the Raspberry Pi computer, accessible via the web-based user interface of the SPIRO software.

- While idling between imaging cycles, the stepper motor positions the stage at alternating $45^{\circ}$ to ensure that all plates are evenly exposed to the conditions in the incubator.

\section{Setting up an experiment}

SPIRO was originally designed for imaging Arabidopsis thaliana seeds and seedlings. We provide detailed guidelines for casting agar plates, sterilizing and plating seeds, and adjusting imaging settings in File S2. For potential updates please refer to the SPIRO Assays Repository ${ }^{23}$.

\section{SPIRO assays}

To thoroughly assess the data quality acquired using SPIRO and further enhance applicability of the imaging platform for the plant biology community, we developed complete pipelines for two commonly used phenotyping assays that would greatly benefit from automated imaging: seed germination and root growth assays. The assays comprise image processing steps carried out by designated macro scripts in FIJI $^{24}$ (distribution of ImageJ) and quantitative data processing steps carried out by custom R scripts. Step by step instructions and scripts are provided in Files S4 and S5. Please note that updates are published in the SPIRO Assays Repository ${ }^{23}$. 
396 Each assay starts with pre-processing of SPIRO raw data to combine 397 individual images into time-lapse stack files with a set scale bar. The

398 preprocessed data is then subjected to semi-automated image segmentation 399 with identification of the objects of interest and measurement of their physical 400 parameters, e.g., perimeter, length, and area, at successive time points. The 401 quantitative data is then processed by $\mathrm{R}$ scripts to first ensure data quality and 402 then apply custom-designed algorithms that determine seed size, germination 403 time, root length and perform suitable statistical analyses.

404 The assays were designed to enable applicability for a broad range of 405 experiment layouts and customization for specific uses, thus we introduced 406 several user-guided steps that allow combining seeds or seedlings into groups 407 of interests for analysis, trimming time ranges, renaming samples, removing 408 outliers, etc.

409 Each assay provides the user with graphical and quantitative outputs and 410 suitable statistical analysis of the data. The assay design ensures quick 411 verification of image segmentation accuracy and identification of potential 412 outliers.

413 Furthermore, to make combining data from several experiments for the 414 statistical analysis user-friendly, we developed the SPIRO Assay 415 Customizer ${ }^{25}$. Customizer takes the quantitative data from one or several 416 experiments cleaned by the quality control $\mathrm{R}$ scripts, and provides the user 417 with an intuitive graphical interface for merging experimental data, 418 regrouping, renaming or removing samples.

419 The step-wise design of each assay with outputs at intermediate stages allow 420 the user to choose between relying on the provided algorithms or taking over 421 at any point to perform their own analysis of the data.

423 SPIRO Seed Germination Assay is based on the simple concept that the perimeter of a seed will steadily increase after germination, i.e., the radicle 
425 emergence ${ }^{26}$, took place. Hence, the image segmentation part of the assay

426 detects individual seeds on the photos (Fig. 2A, Movie S2), and tracks changes

427 in the perimeter of each seed within a user-defined time range. The data is then

428 gathered into user-defined groups, e.g., genotypes or treatments, and subjected

429 to a clean-up using a designated R script. After this, for each seed the earliest

430 time point of steady increase in perimeter is detected and identified as the time

431 of germination. The assay is optimized for an imaging frequency of every 30

432 minutes and thus allows tracking minute differences in germination times. To

433 take into account the effect of imbibition on the seed perimeter and also to

434 compensate for the natural variation in Arabidopsis seed sizes, the germination

435 algorithm normalizes perimeter changes for each seed by comparing it to the

436 same seed perimeter averaged over the first five images in the time-lapse data.

437 The significance of difference between mean germination times for the user-

438 selected groups of seeds is then assessed by the Kaplan-Meier test (Fig. 2B).

439 Furthermore, the assay provides information about the size of individual seeds

440 and the results of t-test comparing seed sizes for user-defined groups (Fig.

441 2C). Additionally, we implemented calculation of other germination

442 parameters that might be valuable for the user, such as rate-of-germination

443 curve, time at maximum germination rate; time required to achieve $50 \%$ of

444 total germination efficacy, time required for $50 \%$ of total seeds to germinate

445 (for detailed information see File S4 and the SPIRO Assays repository ${ }^{23}$ ).

446 We verified the robustness of the semi-automated SPIRO Seed Germination

447 Assay by comparing its results with germination time points detected

448 manually on the same imaging data. The comparison of germination time

449 points for 172 seeds revealed that the automated assay provides results similar

450 to manual assessment $\left(R^{2}=0.88\right.$, Fig. 2D). For this experiments, samples

451 preparation and imaging was done according to the instructions provided in

452 the File $\mathbf{S 4}$.

453 While developing the assay we optimized the SPIRO hardware and protocol

454 for seed plating, and introduced seed plating guides that demark positions for

455 placing seeds at optimal distance from each other and plate rims. As a result,

456 when using four $12 \mathrm{~cm}$ square Petri plates, it is possible to detect germination 

made available under aCC-BY 4.0 International license.

457 for up to 2300 seeds in a single experiment. Additionally, we strongly

458 recommend using SPIRO anti-reflection lids to reduce image segmentation

459 artifacts caused by reflections (Fig. 2 in the File S4). 
A

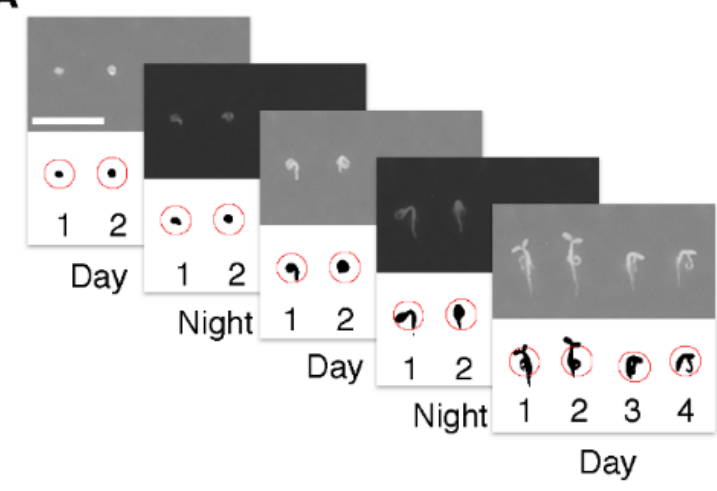

C

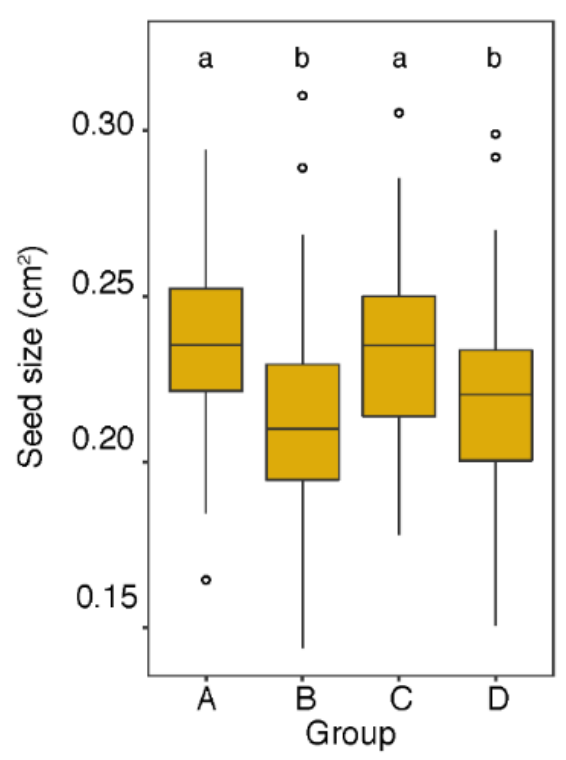

B

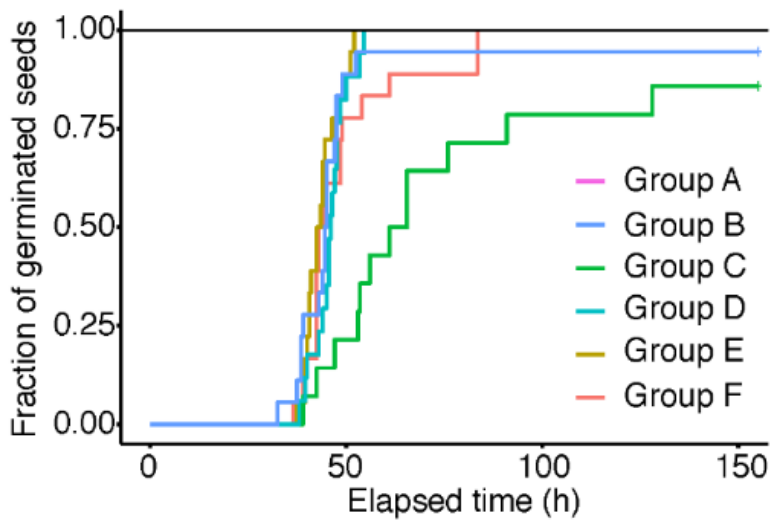

D

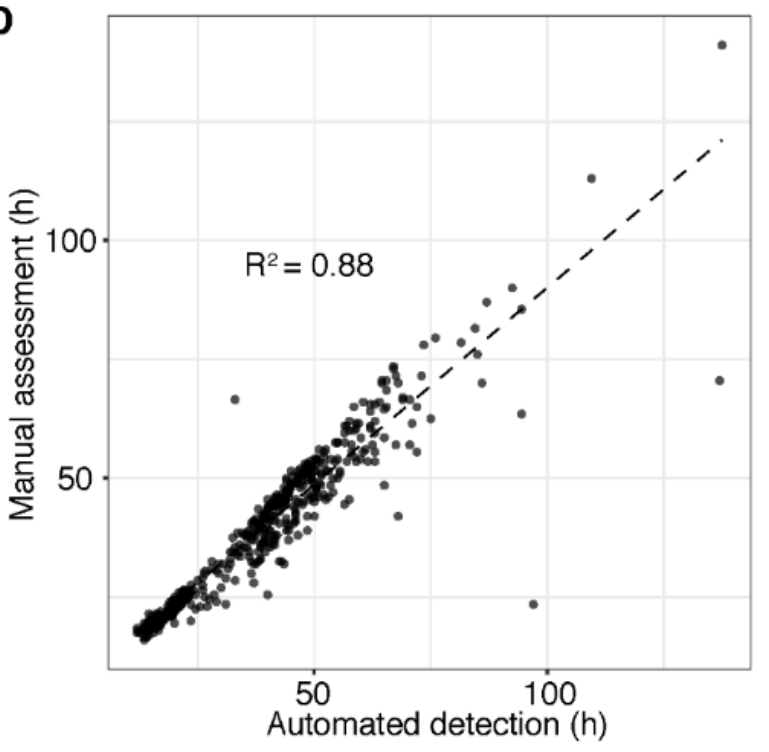

Figure 2. SPIRO Seed Germination Assay.

A. The graphical output of the SPIRO Seed Germination Assay includes a time-lapse stack file, in which each frame contains the original photo and the result of its segmentation, i.e., a mask for each recognized seed annotated by a number and a circle.

B. The assay provides Kaplan-Meier test results for germination of all groups of seeds included into analysis. Additional parameters for germination are calculated using the germinationmetrics package for $\mathrm{R}^{27}$ (for more information see SPIRO Assay manual, File S3).

C. The assay also provides a t-test comparison of the mean seed size for the analyzed groups. Boxes represent interquartile range, the horizontal line denotes the median value, circles indicate outliers. Means of the groups that are not significantly different are annotated with the same small case letters, $n=684$ seeds.

D. Automatic detection of seed germination using SPIRO Seed Germination Assay provides results very similar to manual assessment of the germination $(\mathrm{n}=172)$. 


\section{SPIRO Root Growth Assay}

462 Quantifying primary root length of Arabidopsis seedlings is frequently used

463 as a readout for physiological response to mutations or environmental 464 stimuli ${ }^{13,28,29}$. SPIRO is an excellent platform for seedling root phenotyping.

465 We first tested processing of SPIRO images by existing automated image 466 analysis tools for detection of single roots and root systems on time-lapse 467 data ${ }^{13,30,31}$. As these algorithms were optimized for a certain type of imaging 468 data, their applicability for SPIRO-acquired images was limited.

469 Therefore, we developed the designated SPIRO Root Growth Assay, which 470 uses SPIRO time-lapse data to track primary root length for individual 471 seedlings starting from the germination time-point of the corresponding seed

472 (Fig. 3A, Movie S2), builds a root growth rate model for user-defined groups 473 of seedlings (Fig. 3B), and then performs statistical analysis comparing root 474 lengths and growth rates for the groups (Fig. 3C, File S4, and the SPIRO 475 Assays repository ${ }^{23}$ ). Similar to the SPIRO Seed Germination Assay, the Root 476 Growth Assay provides the user with a graphical output that show the results 477 of image segmentation for each user-selected group (Fig. 3A) and a 478 quantitative output. The latter comprises (i) measurements of the segmented 479 objects performed by the ImageJ macro; (ii) the measurements data cleaned 480 up using a designated R script; (iii) germination time detected for each seed; 481 (iv) curve charts for seedling's root lengths plotted vs absolute time or 482 normalized to individual germination times; (v) curve charts generated by 483 models for root growth rates of user-selected groups of seedlings (Fig. 3B); 484 (vi) bar charts showing predicted root lengths for the groups of seedlings at 485 24-h intervals (Fig. 3C) and (vii) results of the statistical analysis comparing 486 growth rates and root lengths between user-defined groups. For more details 487 please refer to the assay manual in File $\mathbf{S} 4$ and the SPIRO Assays repository ${ }^{23}$.

488 Comparison of manual and automated measurements of 141 Arabidopsis 489 roots, revealed that the SPIRO Root Growth Assay provides accuracy 490 comparable with human performance (Fig. 3D). 

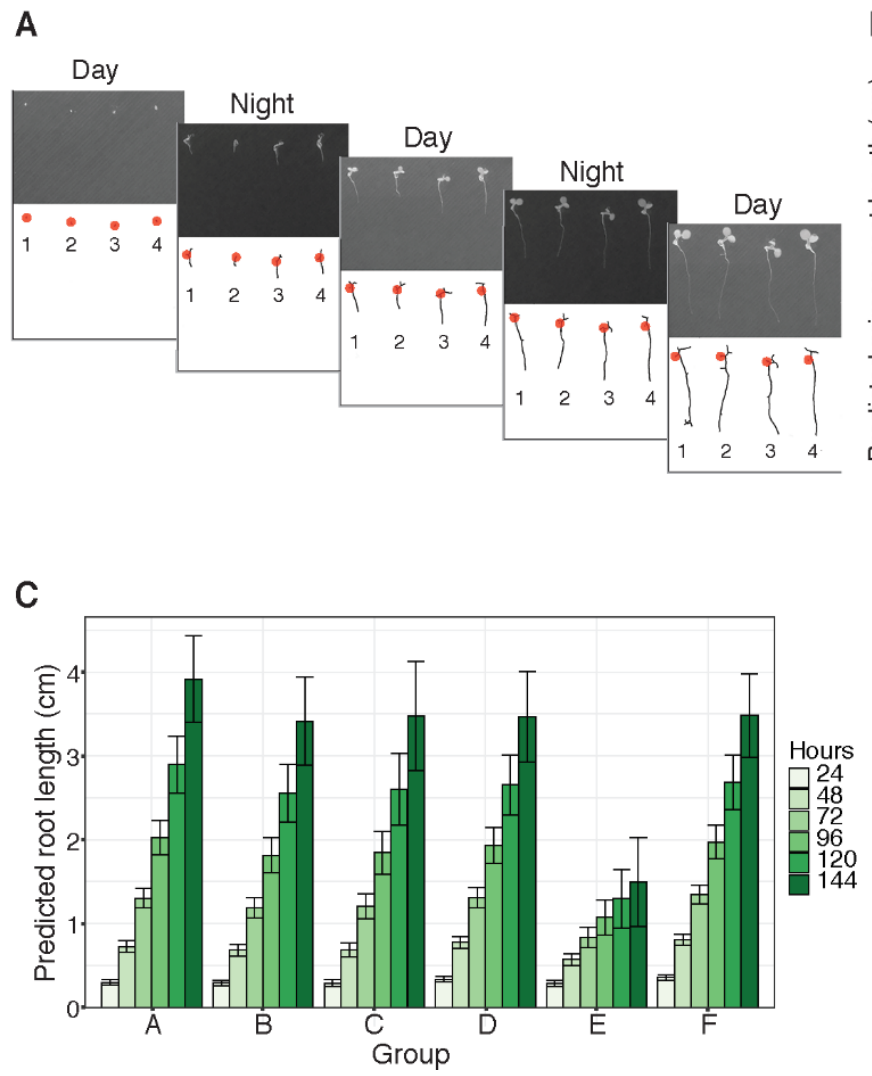

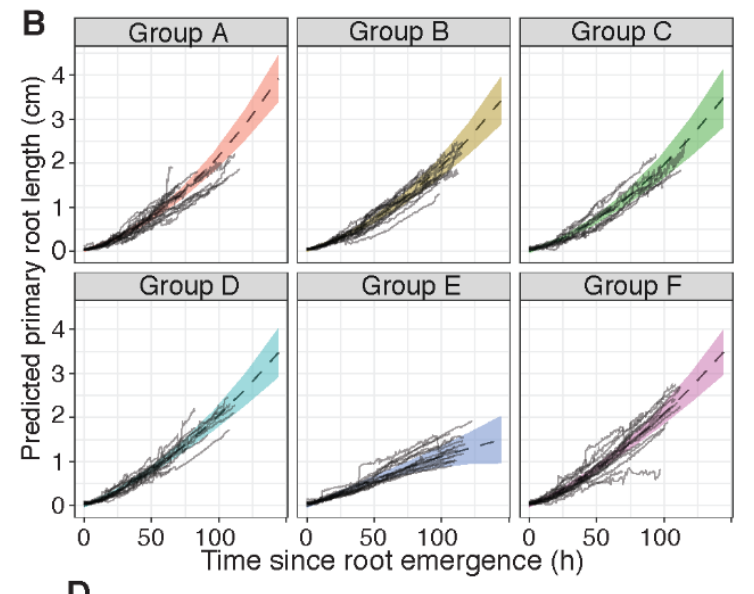

D

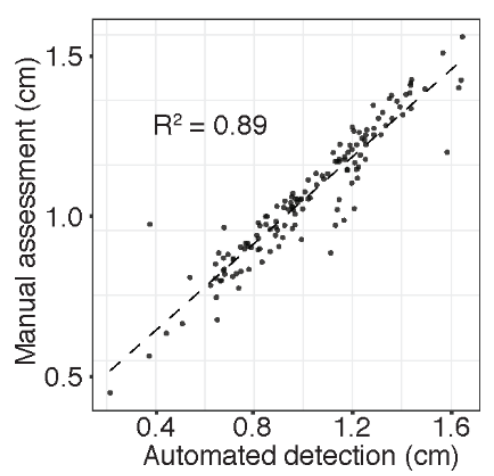

Figure 3. SPIRO Root Growth Assay.

A. Graphical output of the SPIRO Root Growth Assay includes a time-lapse stack file, wherein each frame contains the original photo and the result of its segmentation, i.e., a skeletonized mask for each recognized seedling with denoted root start coordinate (red dot) annotated with a seedling number.

B. The assay builds a model for root growth of each analyzed group (for more information see SPIRO Assay manual, File S4). For each root, the elapsed time is calculated from the time of germination for the corresponding seed. Black solid lines indicate root lengths plotted vs time for each seedling, the dotted black line is the predicted root length, and the colored area indicates standard error.

C. Based on the models shown in B, the assay also predicts average root length for each group at $24 \mathrm{~h}$ intervals. Error bars show standard error.

D. Automatic detection of root length using SPIRO Root Growth Assay provides results very similar to manual assessment of the root lengths ( $\mathrm{n}=141$ seedlings). Manual assessment was done using segmented line tool in ImageJ. 


\section{Discussion}

493 In this publication we provide a thorough description of how to build and use

494 the Smart Plate Imaging Robot, SPIRO. SPIRO provides excellent quality of

495 images, acquired under desired growth conditions at regular time intervals. It

496 is an affordable, versatile and robust imaging platform optimal for every-day

497 use in plant biology and microbiology.

498 Open source engineering solutions can have a great impact on improving the

499 quality, reproducibility, and transparency of research ${ }^{6}$ but the benefit of their

500 implementation is habitually undervalued. For instance, the amount of work

501 hours required for manual time-lapse imaging of Petri plates is often

502 underestimated by calculating only the time needed for acquiring images.

503 However, the daily chore of photographing or scanning plates interferes with

504 other work tasks that have to be fitted between imaging sessions and,

505 depending on the desired imaging frequency, can become quite stressful.

506 SPIRO not only frees up user's time by taking over the imaging workload, it

507 also improves the quality of the acquired data and provides remote access to it

508 from anywhere in the world. The latter feature turned out to be surprisingly

509 useful during COVID-19 lockdown regulations.

510 We strongly believe that reducing the barrier of entry is crucial to the adoption

511 of open science hardware, especially in non-engineering disciplines. In our

512 experience, one of the significant bottlenecks in implementing engineering

513 solutions custom-designed for laboratory use is the requirement of expertise

514 in subjects that are not necessarily popular among biologists, such as

515 mechanical engineering and electronics. SPIRO was developed specifically

516 for biologists, and its design has at its core the concept of being simple and

517 intuitive enough to be assembled and operated with no training in engineering,

518 3D printing, programming or using the SPIRO per se. Furthermore, we strived

519 to develop an imaging platform that can be built anywhere in the world without

520 requiring access to rare specialized infrastructure and would be affordable also

521 for research groups with limited funding. During preparation of this

522 manuscript, multiple SPIROs have been already constructed in six laboratories

523 located in four different countries using only the provided instructions, thus 
524 confirming the need for such platform and validating our approach to making

525 its construction accessible.

526 The current configuration of SPIRO is optimized for image acquisition under

527 conditions commonly used in plant biology and microbiology. Moreover, the

528 underlying Raspberry Pi platform is ideal for further expanding the system. A

529 large variety of Raspberry Pi-compatible sensors and other input/output

530 modules could be incorporated into a custom-built SPIRO system to

531 accommodate different research needs. For example, cheap sensors for

532 temperature and humidity ${ }^{18}$ can be connected to unused general-purpose

533 input/output (GPIO) pins on the computer board. Such upgrades can be

534 valuable when using SPIRO in a growth chamber that does not provide control

535 or logging of these parameters.

536 We based our design on the use of 3D-printed structural components to further

537 facilitate customization of SPIRO. We provide F3D model files that can be

538 easily modified using Autodesk Fusion 360 software (File S1). As of 2021,

539 Autodesk offers a free license tier for academic users, which includes training

540 material. Furthermore, use of 3D-printed parts warrants reproducibility and

541 robustness of the structural components, enables their easy replacement for

542 maintenance and ensures that assembly can be done without access to rare

543 specialized infrastructures. For instance, not only 3D printers are becoming

544 increasingly affordable, with the cheapest models costing less than $€ 200$, but

545 prints can also be ordered using commercial services ${ }^{17}$ or at public Makerspace

546 facilities ${ }^{16}$.

547 We demonstrate analysis of high-throughput SPIRO-acquired data in the two

548 semi-automated assays for seed germination and seedling root growth. The

549 detailed protocols for the assays comprise the complete procedure from

550 preparing Petri plates to statistical analysis of the data. Both assays provide

551 data analysis accuracy closely matching human performance (Fig. 2D and

552 3D). Moreover, the SPIRO Seed Germination Assay enables very high

553 temporal resolution enabling the detection of minute differences in

554 germination times. Importantly, despite its small footprint, the platform still

555 provides practical throughput: a single SPIRO can image up to 2300 seeds in 
556 a single experiment for the Germination Assay, and 190 seedlings for the Root

557 Growth Assay. In their current versions, the assays are implemented using

558 ImageJ and R, software commonly used in biology labs, which should make

559 their use and customization relatively uncomplicated. We have developed

560 them as "plug-and-play" image analyses that are optimized for SPIRO data

561 and provide outstanding results for the intended purpose. Implementation of

562 the recent advances in machine learning approaches ${ }^{32-34}$ will require thorough

563 training of appropriate models for image segmentation, but will eventually

564 enable a more advanced analyses of SPIRO-acquired data such as detangling

565 of crossing roots, measurement of lateral roots, hypocotyls and cotyledons

566 under day and night conditions.

567 The demand for affordable automated platform for Petri plate imaging is

568 clearly illustrated by the number of publications describing various prototypes

569 of such system $7,9,12,32,33,35$. However, we could not find a solution that would

570 be simultaneously affordable, compatible with standard growth cabinets,

571 provide high-quality data and come with instructions comprehensible for a

572 person not trained in engineering. Hence, we pursued developing SPIRO to

573 establish a platform that would enable universal access to automated high-

574 quality imaging for all research groups, independent on their training or

575 funding situation, and enable easy integration of the automated approach into

576 ongoing research.

577 For the sake of posterity, the current version of the complete information about

578 SPIRO's construction and use is provided in the supplementary information of

579 this publication. However, SPIRO is under active development, and all

580 updates are made available on the designated GitHub repositories ${ }^{15,22,23,25}$. We

581 release all components of SPIRO as open-source material under licenses

582 allowing unlimited use and modifications as long as proper attribution is

583 present. The assays provided here are only two examples of the broad variety

584 of possible SPIRO applications. The outstanding quality and fidelity of

585 SPIRO's images forms an excellent basis for any Petri plate-based imaging

586 assay. We hope that SPIRO will help alleviate some pains of routine lab work

587 and will also become a stepping stone for advancement of users' interests in

588 developing further solutions. We encourage users to further customize the 
bioRxiv preprint doi: https://doi.org/10.1101/2021.03.15.435343; this version posted March 24,2021 . The copyright holder for this preprint (which was not certified by peer review) is the author/funder, who has granted bioRxiv a license to display the preprint in perpetuity. It is made available under aCC-BY 4.0 International license.

589 platform, develop image-analysis pipelines suited for their own research and

590 share optimization with the scientific community. 


\section{Competing interests}

592 The authors declare no competing interests.

\section{Acknowledgements}

594 We would like to express our deepest gratitude to: Sebastian Mai (Heidelberg

595 University) who provided valuable advice, resistors and terminal ports for

596 SPIRO hardware development; all members of Prof. Karin Schumacher's

597 research group (Heidelberg University, Germany) for their patience and moral

598 support and especially to Rainer Waadt for the name SPIRO; August Bergh,

599 Thomas Danielsson, and Ping Wu (Ångström laboratory, Uppsala University,

600 Sweden) for their contribution to the development of the early prototype; and

601 to Johan Roxendal (Gothenburg University) and Dan Rosén (Uppsala

602 University) for their contributions to the SPIRO software.

603 This study was supported by the funding from EU Horizon 2020 MSCA IF

604 (799433 to EA Minina), Carl Tryggers Foundation (CTS 14326 and 20:287

605 to EA Minina), DFG (CRC1101, TPA02 to K Schumacher), Formas (2019-

60601565 to AN Dauphinee), Formas (2016-20031 to M Sandgren), Knut and

607 Alice Wallenberg Foundation (2018.0026 to PV Bozhkov), the Swedish

608 Research Council VR (621-2013-4707 to PV Bozhkov), the Swedish

609 Foundation for Strategic Research (Oil Crops for the Future), and Crops for

610 the Future Research Programme at the Swedish University of Agricultural

611 Sciences. 


\begin{tabular}{|c|c|c|}
\hline Supplementary material & File name & Description \\
\hline Table S1 & $\begin{array}{l}\text { Table S1. List of components to } \\
\text { be purchased.pdf }\end{array}$ & $\begin{array}{l}\text { List of components to be purchased } \\
\text { and suggestions of vendors }\end{array}$ \\
\hline Table S2 & $\begin{array}{l}\text { Table S2. List of 3D-printable } \\
\text { components.pdf }\end{array}$ & $\begin{array}{l}\text { List of 3D-printable components and } \\
\text { instructions for printing settings }\end{array}$ \\
\hline Supplementary File 1 & Files for 3D printing.zip & $\begin{array}{l}\text { Printable (STL, 3MF), and editable } \\
\text { (F3D) model files }\end{array}$ \\
\hline Supplementary File 2 & $\begin{array}{l}\text { SPIRO Assembly } \\
\text { Instructions.pdf }\end{array}$ & $\begin{array}{l}\text { SPIRO Assembly Instructions and } \\
\text { links to tutorial videos }\end{array}$ \\
\hline Supplementary File 3 & spiro-software.zip & $\begin{array}{l}\text { SPIRO Software GitHub repository } \\
\text { archive file }\end{array}$ \\
\hline Supplementary File 4 & SPIRO Assays Manual.pdf & SPIRO Assays Manual \\
\hline Supplementary File 5 & SPIRO Assays.zip & $\begin{array}{l}\text { SPIRO Assays GitHub repository } \\
\text { archive file }\end{array}$ \\
\hline Supplementary File 6 & SPIRO Assay Customizer.zip & $\begin{array}{l}\text { SPIRO Assay Customizer archive } \\
\text { file }\end{array}$ \\
\hline Supplementary Video 1 & Supplementary movie 1.mp4 & $\begin{array}{l}\text { SPIRO Overview. A video showing } \\
\text { two SPIRO systems placed in a } \\
\text { plant growth incubator and the time- } \\
\text { lapse video recorded using them }\end{array}$ \\
\hline Supplementary Video 2 & Supplementary movie 2.mp4 & $\begin{array}{l}\text { Demonstration of the image quality } \\
\text { acquired by SPIRO and the } \\
\text { capabilities of the two companion } \\
\text { SPIRO Assays. The movie shows } \\
\text { raw and preprocessed data, as well } \\
\text { as illustrates the output of the } \\
\text { Germination and Root Growth } \\
\text { Assays. }\end{array}$ \\
\hline
\end{tabular}




\section{References}

1. Quint, M. et al. Molecular and genetic control of plant thermomorphogenesis. Nat. Plants 2, 1-9 (2016).

2. Topham, A. T. et al. Temperature variability is integrated by a spatially embedded decision-making center to break dormancy in Arabidopsis seeds. Proc. Natl. Acad. Sci. U. S. A. 114, 6629-6634 (2017).

3. De Ligne, L. et al. Analysis of spatio-temporal fungal growth dynamics under different environmental conditions. IMA Fungus 10, $1-13$ (2019).

4. Pearce, J. M. Return on investment for open source scientific hardware development. Sci. Public Policy 43, 192-195 (2016).

5. Maia Chagas, A. Haves and have nots must find a better way: The case for open scientific hardware. PLoS Biol. 16, e3000014 (2018).

6. Pearce, J. M. Economic savings for scientific free and open source technology: A review. HardwareX 8, e00139 (2020).

7. Yazdanbakhsh, N. \& Fisahn, J. High throughput phenotyping of root growth dynamics, lateral root formation, root architecture and root hair development enabled by PlaRoM. Funct. Plant Biol. 36, 938946 (2009).

8. Subramanian, R., Spalding, E. P. \& Ferrier, N. J. A high throughput robot system for machine vision based plant phenotype studies. Mach. Vis. Appl. 24, 619-636 (2013).

9. Nagel, K. A. et al. The platform GrowScreen-Agar enables identification of phenotypic diversity in root and shoot growth traits of agar grown plants. Plant Methods 16, 1-17 (2020).

10. https://800ezmicro.com/equipment/anaerobic-microaerobicsystems/32-petrifoto-plate-imaging-system.html.

11. Tovar, J. C. et al. Raspberry Pi-powered imaging for plant phenotyping. Appl. Plant Sci. 6, e1031 (2018).

12. Slovak, R. et al. A scalable open-source pipeline for large-scale root phenotyping of Arabidopsis. Plant Cell 26, 2390-2403 (2014).

13. Satbhai, S. B., Göschl, C. \& Busch, W. Automated high-throughput root phenotyping of Arabidopsis thaliana under nutrient deficiency conditions. Methods Mol. Biol. 1610, 135-153 (2017).

14. Https://www.raspberrypi.org/. No Title. 
15. https://github.com/AlyonaMinina/SPIRO.Hardware.

16. https://www.makerspaces.com/what-is-a-makerspace/.

17. https://www.3dhubs.com/.

18. https://tutorials-raspberrypi.com/raspberry-pi-sensors-overview-50important-components/.

19. Eriksson, M. E. \& Millar, A. J. The circadian clock. A plant's best friend in a spinning world. Plant Physiol. 132, 732-738 (2003).

20. Wientjes, E., Philippi, J., Borst, J. W. \& van Amerongen, H. Imaging the Photosystem I/Photosystem II chlorophyll ratio inside the leaf. Biochim. Biophys. Acta - Bioenerg. 1858, 259-265 (2017).

21. Folta, K. M. \& Maruhnich, S. A. Green light: A signal to slow down or stop. J. Exp. Bot. 58, 3099-3111 (2007).

22. https://github.com/jonasoh/spiro.

23. https://github.com/jiaxuanleong/SPIRO.Assays.

24. Schindelin, J. et al. Fiji: An open-source platform for biologicalimage analysis. Nat. Methods 9, 676-682 (2012).

25. https://github.com/jonasoh/spiro-assay-customizer.

26. Bewley, J. D. Seed Germination and Dormancy. Plant Cell 44, 10551066 (1997).

27. Aravind, J., Vimala Devi, S., Radhamani, J., Jacob, S. R. \& Kalyani, S. Germinationmetrics: Seed Germination Indices and Curve Fitting. $\mathrm{R}$ package version 0.1.3.9000. (2020).

28. Patterson, K. et al. Nitrate-regulated glutaredoxins control arabidopsis primary root growth. Plant Physiol. 170, 989-999 (2016).

29. Lucas, M. et al. SHORT-ROOT regulates primary, lateral, and adventitious root development in Arabidopsis. Plant Physiol. 155, 384-398 (2011).

30. Betegón-Putze, I., González, A., Sevillano, X., Blasco-Escámez, D. \& Caño-Delgado, A. I. MyROOT: a method and software for the semiautomatic measurement of primary root length in Arabidopsis seedlings. Plant J. 98, 1145-1156 (2019).

31. Lobet, G., Draye, X. \& Périlleux, C. An online database for plant image analysis software tools. Plant Methods 9, (2013). 
32. Gaggion, N. et al. ChronoRoot: High-throughput phenotyping by deep segmentation networks reveals novel temporal parameters of plant root system architecture. bioRxiv (2020) doi:10.1101/2020.10.27.350553.

33. Yasrab, R. et al. RootNav 2.0: Deep learning for automatic navigation of complex plant root architectures. Gigascience 8, 1-16 (2019).

34. Dobos, O., Horvath, P., Nagy, F., Danka, T. \& Viczián, A. A deep learning-based approach for high-throughput hypocotyl phenotyping. Plant Physiol. 181, 1415-1424 (2019).

35. Ding, X., Vogel, M., Boschke, E., Bley, T. \& Lenk, F. PetriJet Platform Technology: An Automated Platform for Culture Dish Handling and Monitoring of the Contents. J. Lab. Autom. 20, 447456 (2015). 